\title{
The absence of pathological myofibre disarray in the diabetic heart: is it a paradox?
}

\author{
Craig Steven McLACHLAN ${ }^{1}$, PhD, MPH; Shamima LASKER ${ }^{2}$, MSc; Shah M. KERAMAT Ali ${ }^{3}$, DPH, \\ PhD; Lexin WANG ${ }^{4}$, MD, PhD; Herbert JELINEK ${ }^{2}$, PhD \\ ${ }^{1}$ Department of Medical and Molecular Biosciences, University Technology Sydney, NSW, Australia; \\ ${ }^{2}$ School of Community Health, Charles Sturt University, Australia; ${ }^{3}$ Institute of Nutrition and Food \\ science, University of Dhaka, Bangladesh; ${ }^{4}$ School of Biomedical Science, Charles Sturt University, \\ Australia.
}

\begin{abstract}
Myofibre disarray in progressive hypertrophic cardiomyopathy (HCM) is a well established pathological cardiac tissue change and thereby represents a biomarker for that condition. On the other hand, in diabetic cardiomyopathy, myofibre disarray historically has been reported not to occur.This is surprising given that many of the pathological, remodelling and mechanical changes that present in the diabetic ventricle are also present in HCM, for example, myocardial stiffness, myocardial hypertrophy, apoptosis, cell slippage, extensive collagen expression and fibrosis. The question therefore begs is the absence of myocyte disarray in the diabetic heart a paradox or simply an oversight?
\end{abstract}

Keywords: myofibre disarray - diabetic heart - paradox - hypertrophy.

Focal myocyte disarray is a common structural abnormality observed in many cardiac diseases, including coronary heart disease, cor pulmonale, and dilated cardiomyopathy ${ }^{1}$. Specifically myocyte disarray is defined as an area of myocardium where adjacent myocardial cells are aligned perpendicularly or obliquely to each other rather than its normal parallel alignment ${ }^{1}$. Myofibre disarray in patients with hypertrophic cardiomyopathy (HCM) is present in the interventricular septum, which commonly displays greater hypertrophy than the free walls, with $25 \%$ or more of the wall disarrayed ${ }^{1}$.

No reported accounts of myofibre disarray in diabetic cardiomyopathy can be found despite significant pathology similar to HCM. Diabetic cardiomyopathy can occur in the absence of coronary atherosclerosis, hypertension or any other known cardiac disease. However, the coexistence of myocardial ischaemia, hypertension, and diabetic cardiomyopathy, while independent, contributes to the biochemical, anatomic, and functional alterations in cardiac cells and tissues that impair cardiac function. Noninsulin-dependent

Address for correspondence: Dr. Craig McLachlan, Department of Medical and Molecular Bio-Sciences, University of Technology Sydney, NSW, Australia. E-mail: reperfusion@hotmail.com

Received 26 May 2008; accepted for publication 24 July 2008. diabetes induces pathological changes that include increased left ventricular (LV) mass and wall thickness, reduced LV systolic chamber and myocardial function, and increased arterial stiffness ${ }^{2}$. Similar pathological cardiac changes can also be found in the insulin dependent diabetic ${ }^{3}$.

Cardiac diffusion MRI has been applied to map in vivo myocardial fibre architecture. By acquiring registered diffusion and strain MRI images in vivo on both normal and HCM hearts, it has been possible to determine the relationship between myofibre disarray and systolic strain components in HCM, including radial, fibre, and crossfibre strains ${ }^{4}$. For example, myofibre disarray in HCM is correlated with abnormalities of both passive and active myocardial function. This is consistent with the hypothesis that the principal feature of HCM is a reduction in myocardial passive compliance $^{4}$.

Changes in passive compliance are a consequence of diastolic dysfunction in the early disease process in most diabetic hearts 5 . Diastolic dysfunction refers to a condition in which abnormalities in mechanical function are present during diastole. The causes of diastolic dysfunction may be subdivided into a decrease in passive myocardial diastolic compliance, and impairment in active LV relaxation ${ }^{6}$.

Passive compliance can be significantly affected in the asymptomatic diabetic heart ${ }^{7}$. For example, Doppler 
echocardiograms of the mitral valve recorded along with electrocardiograms reveal that components of the total velocity-time integral in the early passive period of ventricular filling and the association with late active period of atrial emptying are depressed in paediatric diabetic patients compared to non-diabetic patients ${ }^{8}$.

Given that passive compliance dysfunction has been shown to be associated with myofibre disarray in HCM this too would suggest the possibility that myofibre disarray could be present in the diabetic heart. In summary, hypertrophic cardiomyopathy is characterized by impaired diastolic function with abnormal stiffness and prolonged relaxation ${ }^{8}$. Likewise it is known that excessive diastolic left ventricular stiffness is an important contributor to heart failure in patients with diabetes mellitus ${ }^{9}$. Diabetes is presumed to increase stiffness through myocardial deposition of collagen and advanced glycation end products (AGEs) ${ }^{9}$. Cardiomyocyte resting tension also elevates stiffness, especially in heart failure with normal left ventricular ejection fraction (LVEF). Cardiac autopsy samples from hypertrophic cardiomyopathy patients; reveal electron microscopic images for three-dimensional disarray, fundamentally constituted from a ring formation of myofibre branches ${ }^{10}$. These myofibre branch changes are likely to promote cardiac muscle stiffness ${ }^{10}$. Whether in the diabetic heart stiffness is promoted also from disarray remains to be identified, what we do know is that excessive collagen, as mentioned above, contributes to a ventricular muscle stiffened state ${ }^{9}$. Indeed both excessive laying down of collagen between fibre strands and the presence of myofibre disarray are well known to coexist in idiopathic cardiomyopathy ${ }^{11}$, once again yet to be determined in the diabetic heart.

\section{References}

1. van der Bel-Kahn J. Muscle fiber disarray in common heart diseases. Am J Cardiol 1977; 40: 355-64.

2. Devereux RB, Roman MJ, Paranicas M, O'Grady MJ, Lee ET, Welty TK, Fabsitz RR, Robbins D, Rhoades ER, Howard BV Impact of diabetes on cardiac structure and function: the strong heart study. Circulation 2000; 101: 2271-6.

3. de Simone G, Mureddu GF, Vaccaro O, Greco R, Sacco M, Rivellese A, Contaldo F, Riccardi G. Cardiac abnormalities in type 1 diabetes. Ital Heart $J$ 2000; 1: 493-9.

4. Tseng WY, Dou J, Reese TG, Wedeen VJ. Imaging myocardial fiber disarray and intramural strain hypokinesis in hypertrophic cardiomyopathy with MRI. J Magn Reson Imaging 2006; 23: 1-8.

5. Wang J, Song Y, Wang Q, Kralik PM, Epstein PN. Causes and characteristics of diabetic cardiomyopathy. Rev Diabet Stud 2006; 3: 108-17.

6. Gilbert JC, Glantz SA. Determinants of LV filling and of the diastolic pressure-volume relation. Circ Res 1989, 64: 827-52.

7. Fein FS, Sonnenblick EH. Diabetic cardiomyopathy Cardiovasc Drugs Ther 1994; 8: 65-73.

8. Kato T, Noda A, Izawa H, Nishizawa T, Somura F, Yamada A, Nagata K, Iwase M, Nakao A, Yokota M. Myocardial velocity gradient as a noninvasively determined index of left ventricular diastolic dysfunction in patients with hypertrophic cardiomyopathy. J Am Coll Cardiol 2003; 42: 278-85.

9. Cosson S, Kevorkian JP. Left ventricular diastolic dysfunction: an early sign of diabetic cardiomyopathy? Diabetes Metab 2003; 29: 455-66.

10. Izumi T. Myofiber branching in idiopathic cardiomyopathy under the scanning electron microscope. Jpn Circ J 1982; 46: 443-9.

11. Noda S. Histopathology of endomyocardial biopsies from patients with idiopathic cardiomyopathy; quantitative evaluation based on multivariate statistical analysis. Jpn Circ J 1980; 44: 95-116.

Conflict of interest: none declared. 